\title{
DERIVING VON KOCH'S INEQUALITY WITHOUT USING THE RIEMANN HYPOTHESIS.
}

\author{
SEIJI Fujino ${ }^{\dagger} 1$
}

2021.Nov.23

\begin{abstract}
In this paper, we derive Von Koch's inequality without using the Riemann Hypothesis. As well known, Von Koch's inequality is equivalent to the Riemann Hypothesis. Namely, we show that the Riemann Hypothesis is true.
\end{abstract}

\section{Deriving Von Koch's inequality without using the Riemann \\ HYPOTHESIS.}

\subsection{Introduction.}

The purpose of this paper is to derive that Von Koch's inequality without using the Riemann Hypothesis. As well known, Von Koch's inequality is equivalent to the Riemann Hypothesis. Namely, we show that the Riemann Hypothesis is true. The proof steps are as follows :

1.1.1. First, we define the functions $R_{\alpha}^{ \pm}(x)$ as follows :

$$
\frac{1}{x R_{\alpha}^{ \pm}(x)}=\frac{e}{\sqrt{2 \pi} \alpha}\left(1 \pm \frac{1}{e x}\right)
$$

where $x, \alpha$ are positive real numbers. We prove that there exist a constant $C>$ $1(\in \mathbb{R})$ such that for all sufficiently large $x>1$ satisfies that the inequality :

$$
|\pi(x)-\operatorname{li}(x)| \leq C\left(\operatorname{li}(x)-\frac{x}{\log (x)}\right) .
$$

1.1.2. Second, we define that the distribution function :

$$
\Pi_{\alpha}^{ \pm}(x):=x\left(\exp \left(\frac{1}{\log (x) R_{\alpha}^{ \pm}(\log (x))}\right)\right)
$$

Thereby, we derive that for sufficiently large $x>1$, the following inequalities are satisfied :

$$
\begin{aligned}
\frac{x}{\log (x)} & \leq \Pi_{\alpha}^{+}(x), \\
x\left(\frac{1}{\log (x)}\right)^{\prime} & \leq x\left(\frac{\Pi_{\alpha}^{+}(x)}{x}\right)^{\prime},
\end{aligned}
$$

where a positive real number $\alpha>0$ is constant.

1991 Mathematics Subject Classification. Primary 11N05; Secondary 11A41.

Key words and phrases. Number theory, Von Koch's inequality, Riemann Hypothesis.

${ }^{1} \uparrow$ RHC institute. No.702, Shinjuku-Komuro-BLD.,4-1-22 Shinjuku,Shinjuku-ku,Tokyo,Japan. 160-0022, xfujino001@gmail.com 
2 DERIVING VON KOCH'S INEQUALITY WITHOUT USING THE RIEMANN HYPOTHESIS.

1.1.3. Third, using these results above, we derive that there exist a constant $C>$ $1(\in \mathbb{R})$ such that for all sufficiently large real number $x \geq 2(\in \mathbb{R})$, the following conditions are satisfied :

$$
|\pi(x)-\operatorname{li}(x)| \leq C\left(\frac{\sqrt{2 \pi} \alpha}{48}\right)^{\frac{1}{4}} \exp \left(\frac{e}{\sqrt{2 \pi} \alpha}\right) x^{\frac{1}{\sqrt{2 \pi} \alpha}}\left(\frac{1}{\log (x)}\right)^{\frac{3}{4}} \exp \left(\frac{1}{\log (x)}\right),
$$

where a positive real number $\alpha>0$ is constant.

Therefore, we examine that the derivation of Von Koch's inequality that :

$$
|\pi(x)-\operatorname{li}(x)| \leq O\left(x^{\frac{1}{2}} \log (x)\right) .
$$

Namely, we show that the Riemann Hypothesis is true.

1.1.4. Fourth, additionally, we obtain the following result :

$$
|\pi(x)-\operatorname{li}(x)| \leq O\left(x^{\frac{1}{e}} \log (x)\right) .
$$

We further raise the following problem :

$$
e=\sup \left\{d>1|| \pi(x)-\operatorname{li}(x) \mid \leq O\left(x^{\frac{1}{d}} \log (x)\right)\right\} .
$$

We give details of the discussions as follows.

\subsection{Definition of functions and symbols.}

We first give some definitions and symbols used in this paper as follows.

Definition 1.1. Let $x>1$ be a positive real number $(x \in \mathbb{R})$.

$$
\begin{aligned}
\pi(x) & :=\sum_{\substack{p \leq x \\
p: p r i m e}} 1 \\
& : \text { the number of prime numbers less than or equal to } x . \\
\log (x) & :=\log _{e}(x) \\
& : \log \text { is as natural logarithm, e is Napier number. } \\
\operatorname{li}(x) & :=\lim _{\epsilon \rightarrow 0}\left(\int_{0}^{1-\epsilon}+\int_{1+\epsilon}^{x}\right) \frac{d t}{\log (t)} \\
& : \text { logarithmic integral function. }
\end{aligned}
$$

Definition 1.2. Let $\alpha>0$ be a positive real number $(\in \mathbb{R})$. For all positive real number $x>1(\in \mathbb{R})$, the functions $R_{\alpha}^{+}(x)$ and $R_{\alpha}^{-}(x)$ are defined as follows:

$$
\begin{aligned}
& R_{\alpha}^{+}(x):=\frac{\sqrt{2 \pi} \alpha}{e x+1} \\
& R_{\alpha}^{-}(x):=\frac{\sqrt{2 \pi} \alpha}{e x-1}
\end{aligned}
$$

The function $R_{\alpha}^{ \pm}(x)$ are combined $R_{\alpha}^{+}(x)$ and $R_{\alpha}^{-}(x)$ as follows:

$$
R_{\alpha}^{ \pm}(x):=\frac{\sqrt{2 \pi} \alpha}{e x \pm 1}
$$

Therefore, the following equation is satisfied: (Refer to Feynman[2])

$$
\frac{1}{x R_{\alpha}^{ \pm}(x)}=\frac{e}{\sqrt{2 \pi} \alpha}\left(1 \pm \frac{1}{e x}\right)
$$


DERIVING VON KOCH'S INEQUALITY WITHOUT USING THE RIEMANN HYPOTHESIS. 3

Lemma 1.3. Let $\alpha>0$ be a positive real number $(\in \mathbb{R})$. For all real number $x>1(\in \mathbb{R})$, the following condition is satisfied :

$$
\operatorname{li}(x) \geq x\left(\frac{\sqrt{2 \pi} \alpha}{e \log (x)}\right)>x\left(\frac{\sqrt{2 \pi} \alpha}{e \log (x)+1}\right)=x R_{\alpha}^{+}(\log (x))
$$

where

$$
\alpha \leq \frac{e}{\sqrt{2 \pi}} \quad(\alpha, x \in \mathbb{R})
$$

Proof. Using Definition(1.2) and apply integration by parts of the logarithmic integral function $\operatorname{li}(x)$. There is a positive integer $n \geq 1$ such that for all real number $x>1$, the following inequalities are satisfied :

$$
\begin{aligned}
\operatorname{li}(x) & =\lim _{\epsilon \rightarrow 0}\left(\int_{0}^{1-\epsilon}+\int_{1+\epsilon}^{x}\right) \frac{d t}{\log (t)} \\
& =\frac{0 ! x}{\log (x)}+\frac{1 ! x}{(\log (x))^{2}}+\cdots+\frac{(n-1) ! x}{(\log (x))^{n}}+\lim _{\epsilon \rightarrow 0}\left(\int_{0}^{1-\epsilon}+\int_{1+\epsilon}^{x}\right) \frac{n d t}{(\log (t))^{n+1}} \\
& \geq x\left(\frac{1}{\log (x)}+\frac{1}{(\log (x))^{2}}+\frac{2}{(\log (x))^{3}}+\frac{6}{(\log (x))^{4}}+\cdots+\frac{(n-1) !}{(\log (x))^{n}}\right) \\
& \geq x\left(\frac{\sqrt{2 \pi} \alpha}{e \log (x)}\right) \\
& \geq x\left(\frac{\sqrt{2 \pi} \alpha}{e \log (x)+1}\right)=x R_{\alpha}^{+}(\log (x))
\end{aligned}
$$

Therefore, the following conditions are satisfied :

$$
\operatorname{li}(x) \geq x\left(\frac{\sqrt{2 \pi} \alpha}{e \log (x)}\right)>x\left(\frac{\sqrt{2 \pi} \alpha}{e \log (x)+1}\right)=x R_{\alpha}^{+}(\log (x))
$$

1.3. The upper and the lower bound of $\pi(x)$.

We know as the result that the upper and the lower bound of $\pi(x)$ exists. (Lemma1.4) Using this result, we show that the upper bound of $|\pi(x)-\operatorname{li}(x)|$ exists. (Lemma1.5)

Lemma 1.4. There exists positive real numbers $C_{1}>1$ and $1>C_{2}>0$ such that for all positive real number $x \geq 2$, the following conditions are satisfied:

$$
C_{2} \frac{x}{\log (x)}<\pi(x)<C_{1} \frac{x}{\log (x)} \quad\left(C_{1}, C_{2}, x \in \mathbb{R}\right)
$$

(Refer to Wladyslaw [1])

Lemma 1.5. There exist a real number $C>1(\in \mathbb{R})$ such that for all sufficiently large real number $x>2(\in \mathbb{R})$, the following inequality is satisfied:

$$
|\pi(x)-\operatorname{li}(x)| \leq C\left(\operatorname{li}(x)-\frac{x}{\log (x)}\right)
$$

Proof. The proof is described in the following section. 
4 DERIVING VON KOCH'S INEQUALITY WITHOUT USING THE RIEMANN HYPOTHESIS.

\subsection{Definition of distribution functions.}

On this subsection, we define distribution functions and prove some lemma for preparing to prove the main theorem(1.9).

Definition 1.6. Let $\alpha>0$ be a positive real number $(\in \mathbb{R})$. For all real number $x>1(\in \mathbb{R})$, the distribution functions $\Pi_{\alpha}^{+}(x)$ and $\Pi_{\alpha}^{-}(x)$ are defined as follows:

$$
\begin{aligned}
& \Pi_{\alpha}^{+}(x):=x \exp \left(\frac{1}{\log (x) R_{\alpha}^{+}(\log (x))}\right) \\
& \Pi_{\alpha}^{-}(x):=x \exp \left(\frac{1}{\log (x) R_{\alpha}^{-}(\log (x))}\right)
\end{aligned}
$$

The distribution function $\Pi_{\alpha}^{ \pm}(x)$ are combined and described by $\Pi_{\alpha}^{+}(x)$ and $\Pi_{\alpha}^{-}(x)$ as follows:

$$
\Pi_{\alpha}^{ \pm}(x):=x \exp \left(\frac{1}{\log (x) R_{\alpha}^{ \pm}(\log (x))}\right)
$$

Lemma 1.7. Let $\alpha>0$ be a positive real number $(\in \mathbb{R})$. For all sufficiently large real number $x>1(\in \mathbb{R})$, the following condition is satisfied :

$$
\frac{x}{\log (x)} \leq \Pi_{\alpha}^{ \pm}(x)
$$

Proof. For all sufficiently large real number $x>1$, the following conditions are satisfied :

$$
\frac{1}{\log (x)} \leq \exp \left(\frac{e}{\sqrt{2 \pi} \alpha}\left(1 \pm \frac{1}{e \log (x)}\right)\right)=\exp \left(\frac{1}{\log (x) R_{\alpha}^{ \pm}(\log (x))}\right)
$$

Therefore, the following condition is satisfied :

$$
\frac{x}{\log (x)} \leq x \exp \left(\frac{1}{\log (x) R_{\alpha}^{ \pm}(\log (x))}\right)
$$

Lemma 1.8. Let $\alpha>0$ be a positive real number $(\in \mathbb{R})$. For all sufficiently large real number $x>1(\in \mathbb{R})$,

$$
x\left(\frac{1}{\log (x)}\right)^{\prime} \leq x\left(\frac{\Pi_{\alpha}^{+}(x)}{x}\right)^{\prime}
$$

where the real number $\alpha>0(\in \mathbb{R})$ is satisfied as follows:

$$
\left.1 \leq \frac{1}{\sqrt{2 \pi} \alpha} \exp \left(\frac{e}{\sqrt{2 \pi} \alpha}\right) \quad \text { that is, } \alpha \leq \frac{e}{\sqrt{2 \pi}}\right)
$$

Proof. For all sufficiently large $x>1$, the following equations are satisfied :

$$
\begin{gathered}
x\left(\frac{1}{\log (x)}\right)^{\prime}=x\left(\frac{1}{x(\log (x))^{2}}\right) \\
x\left(\frac{\Pi_{\alpha}^{+}(x)}{x}\right)^{\prime}=x\left(\exp \left(\frac{e}{\sqrt{2 \pi} \alpha}\left(1+\frac{1}{e \log (x)}\right)\right)\right)^{\prime} \\
=x\left(\frac{1}{\sqrt{2 \pi} \alpha} \frac{1}{x(\log (x))^{2}} \exp \left(\frac{e}{\sqrt{2 \pi} \alpha}\left(1+\frac{1}{e \log (x)}\right)\right)\right)
\end{gathered}
$$


DERIVING VON KOCH'S INEQUALITY WITHOUT USING THE RIEMANN HYPOTHESIS. 5

The right side of each equations(1.31) and (1.32) are divided by $1 /(\log (x))^{2}$. Hence, the following inequality is satisfied :

$$
1 \leq \frac{1}{\sqrt{2 \pi} \alpha} \exp \left(\frac{e}{\sqrt{2 \pi} \alpha}\left(1+\frac{1}{e \log (x)}\right)\right)
$$

Therefore, the following condition is satisfied :

$$
x\left(\frac{1}{\log (x)}\right)^{\prime} \leq x\left(\frac{\Pi_{\alpha}^{+}(x)}{x}\right)^{\prime}
$$

\subsection{Inequalities for evaluating the number of prime numbers.}

We show that the following Theorem(1.9) is satisfied using the result of Lemma(1.5) is satisfied.

Theorem 1.9. Inequalities for evaluating the number of prime numbers (1).

Let $\alpha>0$ be a positive real number $(\in \mathbb{R})$. There exist a positive real number $C>1(\in \mathbb{R})$ such that for all sufficiently large real number $x \geq 2(\in \mathbb{R})$, the following conditions are satisfied:

$$
|\pi(x)-\operatorname{li}(x)| \leq C\left(\frac{\sqrt{2 \pi} \alpha}{48}\right)^{\frac{1}{4}} \exp \left(\frac{e}{\sqrt{2 \pi} \alpha}\right) x^{\frac{1}{\sqrt{2 \pi} \alpha}}\left(\frac{1}{\log (x)}\right)^{\frac{3}{4}} \exp \left(\frac{1}{\log (x)}\right)
$$

where the positive real number $\alpha>0$ is satisfied as follows :

$$
\begin{aligned}
& \left.1 \leq \frac{1}{\sqrt{2 \pi} \alpha} \exp \left(\frac{e}{\sqrt{2 \pi} \alpha}\right) \quad \text { (that is, } \alpha \leq \frac{e}{\sqrt{2 \pi}}\right) \\
& \frac{1}{\sqrt{2 \pi}} \leq \alpha \leq C \frac{e}{\sqrt{2 \pi}} \\
& \exp \left(\frac{e}{\sqrt{2 \pi} \alpha}\right)=\lim _{x \rightarrow \infty} \exp \left(\frac{1}{x R_{\alpha}^{ \pm}(x)}\right)
\end{aligned}
$$

Proof. Using Lemma(1.5), this theorem is proof. The proof is described in the following section.

Corollary 1.10. Inequalities for evaluating the number of prime numbers (2).

There exist a positive real number $C>1(\in \mathbb{R})$ such that for all $\epsilon>0(\in \mathbb{R})$ and for all sufficiently large $x \geq 2(\in \mathbb{R})$, the following conditions are satisfied:

$$
|\pi(x)-\operatorname{li}(x)| \leq C\left(\frac{1}{48}\right)^{\frac{1}{4}} \exp (e) x\left(\frac{1}{\log (x)}\right)^{\frac{3}{4}} \exp \left(\frac{1}{\log (x)}\right)
$$

Proof. Using Theorem(1.9), put a positive real number $\alpha>0$ as follows:

$$
\alpha=\frac{1}{\sqrt{2 \pi}}
$$

Therefore, the inequality(1.39) are satisfied.

The result of Corollary(1.9) is similar to the following result : (Refer to Wladyslaw[1])

$$
(\exists C>0)|\pi(x)-\operatorname{li}(x)| \leq O(x \exp (-C \sqrt{\log (x)}))
$$


6 DERIVING VON KOCH'S INEQUALITY WITHOUT USING THE RIEMANN HYPOTHESIS.

Comparing inequalities(1.39) and (1.41), the following condition is satisfied :

$$
O\left(x\left(\frac{1}{\log (x)}\right)^{\frac{3}{4}} \exp \left(\frac{1}{\log (x)}\right)\right) \leq O(x \exp (-C \sqrt{\log (x)}))
$$

Namely, the asymptotic of (1.39) gives better than that of (1.41).

Therefore, put $\alpha=2 / \sqrt{2 \pi}$. the theorem above are satisfied as follows :

Corollary 1.11. Inequalities for evaluating the number of prime numbers (3). There exist a positive real number $C>1(\in \mathbb{R})$ such that for all $\epsilon>0(\in \mathbb{R})$ and for all sufficiently large $x \geq 2(\in \mathbb{R})$, the following condition is satisfied:

$$
|\pi(x)-\operatorname{li}(x)| \leq C\left(\frac{1}{24}\right)^{\frac{1}{4}} \exp \left(\frac{e}{2}\right) x^{\frac{1}{2}}\left(\frac{1}{\log (x)}\right)^{\frac{3}{4}} \exp \left(\frac{1}{\log (x)}\right)
$$

Proof. Using Theorem(1.9) and the following conditions are satisfied:

$$
1 \leq \frac{1}{\sqrt{2 \pi} \alpha} \exp \left(\frac{e}{\sqrt{2 \pi} \alpha}\right)
$$

Put a positive real number $\alpha>0$ as follows:

$$
\alpha=\frac{2}{\sqrt{2 \pi}}\left(\geq \frac{1}{\sqrt{2 \pi}}\right)
$$

Hence, the following inequalities is satisfied :

$$
\begin{aligned}
1 & \leq \frac{1}{\sqrt{2 \pi} \alpha} \exp \left(\frac{e}{\sqrt{2 \pi} \alpha}\right) \\
& =\frac{1}{\sqrt{2 \pi} \frac{2}{\sqrt{2 \pi}}} \exp \left(\frac{e}{\sqrt{2 \pi} \frac{2}{\sqrt{2 \pi}}}\right) \quad\left(\because \alpha=\frac{2}{\sqrt{2 \pi}}\right) \\
& =\frac{1}{2} \exp \left(\frac{e}{2}\right) \quad(=1.946424 \cdots)
\end{aligned}
$$

The positive real number $\alpha>0$ is satisfied the above conditions of (1.44) and (1.45). Therefore, there exist a positive real number $C>1$ such that for all sufficiently large $x \geq 2$, the following condition is satisfied :

$$
|\pi(x)-\operatorname{li}(x)| \leq C\left(\frac{1}{24}\right)^{\frac{1}{4}} \exp \left(\frac{e}{2}\right) x^{\frac{1}{2}}\left(\frac{1}{\log (x)}\right)^{\frac{3}{4}} \exp \left(\frac{1}{\log (x)}\right)
$$

Furthermore, we obtain the following conclusion.

Corollary 1.12. Von Koch's inequality

$$
(\exists C>1)(\forall \epsilon>0)(\forall x>>2)|\pi(x)-\operatorname{li}(x)| \leq C x^{\frac{1}{2}} \log (x)
$$

where $C, \epsilon, x \in \mathbb{R}$. Namely,

$$
|\pi(x)-\operatorname{li}(x)| \leq O\left(x^{\frac{1}{2}} \log (x)\right)
$$

Proof. Fixed $\epsilon>0$. For all sufficient large $x \geq 2$, the following conditions are satisfied :

$$
\left(\frac{1}{\log (x)}\right)^{\frac{3}{4}} \exp \left(\frac{1}{\log (x)}\right)<\log (x)<x^{\epsilon}
$$


DERIVING VON KOCH'S INEQUALITY WITHOUT USING THE RIEMANN HYPOTHESIS. 7

Therefore,there exist a positive real number $C>0$ such that for all sufficiently large $x \geq 2$, the following inequalities are satisfied :

$$
\begin{aligned}
& |\pi(x)-\operatorname{li}(x)| \\
& \leq C\left(\frac{1}{24}\right)^{\frac{1}{4}} \exp \left(\frac{e}{2}\right) x^{\frac{1}{2}}\left(\frac{1}{\log (x)}\right)^{\frac{3}{4}} \exp \left(\frac{1}{\log (x)}\right) \\
& \leq C x^{\frac{1}{2}} \log (x)
\end{aligned}
$$

Furthermore, Let $\alpha=e / \sqrt{2 \pi}$. the following inequality is satisfied :

Corollary 1.13. The expansion of the inequality that evaluate the number of prime number.

$$
(\exists C>1)(\forall \epsilon>0)(\forall x>>2)|\pi(x)-\operatorname{li}(x)| \leq C x^{\frac{1}{e}} \log (x)
$$

where $C, \epsilon, x \in \mathbb{R}$. Namely,

$$
|\pi(x)-\operatorname{li}(x)| \leq O\left(x^{\frac{1}{e}} \log (x)\right)
$$

Proof. Using theorem(1.9), put a positive real number $\alpha>0$ as follows:

$$
\alpha=\frac{e}{\sqrt{2 \pi}}\left(\geq \frac{e}{\sqrt{2 \pi}}\right)
$$

Thus, the following conditions are satisfied :

$$
\begin{aligned}
1 & \leq \frac{1}{\sqrt{2 \pi} \alpha} \exp \left(\frac{e}{\sqrt{2 \pi} \alpha}\right) \\
& =\frac{1}{\sqrt{2 \pi} \frac{e}{\sqrt{2 \pi}}} \exp \left(\frac{e}{\sqrt{2 \pi} \frac{e}{\sqrt{2 \pi}}}\right) \quad\left(\because \alpha=\frac{e}{\sqrt{2 \pi}}\right) \\
& =\frac{1}{e} \exp (1) \quad(=1)
\end{aligned}
$$

Therefore the following condition is satisfied :

$$
\begin{aligned}
& |\pi(x)-\operatorname{li}(x)| \\
& \leq C\left(\frac{e}{48}\right)^{\frac{1}{4}} \exp (1) x^{\frac{1}{e}}\left(\frac{1}{\log (x)}\right)^{\frac{3}{4}} \exp \left(\frac{1}{\log (x)}\right) \\
& \leq C x^{\frac{1}{e}} \log (x) .
\end{aligned}
$$

We have an problem that the upper limit of the real number $d$ that satisfies the formula $|\pi(x)-\operatorname{li}(x)| \leq O\left(x^{1 / d} \log (x)\right)$ is $e$ correct or not. That is, the following problem can be considered.

Problem 1.14. The upper limit of the inequality that evaluate the number of prime number.

(1.57) $e=\sup \left\{d>1|(\exists C>1)(\forall \epsilon>0)(\forall x>>2)| \pi(x)-\operatorname{li}(x) \mid \leq C x^{\frac{1}{d}} \log (x)\right\}$ where $C, \epsilon, x \in \mathbb{R}$. Namely,

$$
e=\sup \left\{d>1|| \pi(x)-\operatorname{li}(x) \mid \leq O\left(x^{\frac{1}{d}} \log (x)\right)\right\}
$$


8 DERIVING VON KOCH'S INEQUALITY WITHOUT USING THE RIEMANN HYPOTHESIS.

We expect the problem(1.14) to be correct. In future, we try to solve this problem.

\section{The Proof of the Discussion above.}

On this section, we first give the proof of Lemma(1.5) and second Theorem(1.9) as follows.

\subsection{Proof of Lemma(1.5).}

Proof. For sufficiently large $x>2$, the sizes of the number of prime numbers $\pi(x)$ and logarithmic integral function $\operatorname{li}(x)$ are switched infinitely. Namely, the positive and negative of the difference li $(x)-\pi(x)$ are switched infinitely. (Refer to Wladyslaw [1]) Therefore, we consider the following two cases, Case 1) and Case 2), separately :

$$
\begin{aligned}
& \text { Case1) }: \pi(x) \leq \operatorname{li}(x) \\
& \text { Case2) }: \operatorname{li}(x)<\pi(x)
\end{aligned}
$$

We first discuss the case 1) and second the case 2).

Case 1) When the inequality $\pi(x) \leq \operatorname{li}(x)$ is satisfied :

We show that there exist a real number $C>0$ such that for all real number $x>2$ the following condition is satisfied :

$$
\operatorname{li}(x)-\pi(x) \leq C\left(\operatorname{li}(x)-\frac{x}{\log (x)}\right)
$$

Because, using Lemma(1.4), there exist a real number $C_{1}>1$ such that the following conditions are satisfied :

$$
\begin{aligned}
& \operatorname{li}(x)<C_{1} \operatorname{li}(x) \\
& \pi(x)<C_{1} \frac{x}{\log (x)}
\end{aligned}
$$

Subtracting both side of inequality (2.5) from that of inequality $(2.4)$, for all real number $x>2$ the following conditions are satisfied :

$$
\operatorname{li}(x)-\pi(x) \leq C_{1}\left(\operatorname{li}(x)-\frac{x}{\log (x)}\right)
$$

Therefore, put $C=C_{1}$, the following inequality is satisfied :

$$
|\pi(x)-\operatorname{li}(x)| \leq C\left(\operatorname{li}(x)-\frac{x}{\log (x)}\right)
$$

The end of the case 1) proof. We next discuss the case 2).

Case 2) When the inequality $\operatorname{li}(x)<\pi(x)$ is satisfied :

Assume that the inequalitty (1.22) is not satisfied (reductio ad absurdum). Namely, assume that for all $C>0$, there exist a sufficiently large real number $x>2$, the following condition is satisfied :

$$
\pi(x)-\operatorname{li}(x)>C\left(\operatorname{li}(x)-\frac{x}{\log (x)}\right)
$$


DERIVING VON KOCH'S INEQUALITY WITHOUT USING THE RIEMANN HYPOTHESIS. 9

Using Lemma(1.3) and Lemma(1.4), the following conditions are satisfied :

$$
\begin{aligned}
& \pi(x)<C_{1} \frac{x}{\log (x)} \quad(\because \operatorname{Lemma}(1.4)) \\
& x\left(\frac{\sqrt{2 \pi} \alpha}{e \log (x)}\right)<\operatorname{li}(x) \quad(\because \operatorname{Lemma}(1.3))
\end{aligned}
$$

where

$$
0<\alpha \leq \frac{e}{\sqrt{2 \pi}} \quad(\alpha \in \mathbb{R})
$$

Adding the both side of inequalities(2.9) and (2.10), thus the following condition is satisfied :

$$
\pi(x)+x\left(\frac{\sqrt{2 \pi} \alpha}{e \log (x)}\right)<C_{1} \frac{x}{\log (x)}+\operatorname{li}(x)
$$

Transform the inequality(2.12) above, the following condition is satisfied :

$$
\pi(x)-\operatorname{li}(x)<C_{1} \frac{x}{\log (x)}-x\left(\frac{\sqrt{2 \pi} \alpha}{e \log (x)}\right)
$$

Hence, for sufficiently large $x(\geq 2)$, the following inequalities are satisfied :

$$
\begin{aligned}
\pi(x)-\operatorname{li}(x) & <C_{1} \frac{x}{\log (x)}-x\left(\frac{\sqrt{2 \pi} \alpha}{e \log (x)}\right) \\
& <\left(C_{1}-\frac{\sqrt{2 \pi} \alpha}{e}\right) \frac{x}{\log (x)}
\end{aligned}
$$

Therefore, the following conditions are satisfied:

$$
\pi(x)-\operatorname{li}(x)<\left(C_{1}-\frac{\sqrt{2 \pi} \alpha}{e}\right) \frac{x}{\log (x)} .
$$

Because the inequality $\left(C_{1}-\sqrt{2 \pi} \alpha / e\right)>0$ is satisfied, thus the following inequality is satisfied :

$$
C_{1}>\frac{\sqrt{2 \pi} \alpha}{e}
$$

Using the assumption(2.8) and the inequality(2.15), the following inequalities are satisfied :

$$
\begin{aligned}
& 0<C\left(\operatorname{li}(x)-\frac{x}{\log (x)}\right)<\pi(x)-\operatorname{li}(x) \\
& \pi(x)-\operatorname{li}(x)<\left(C_{1}-\frac{\sqrt{2 \pi} \alpha}{e}\right) \frac{x}{\log (x)}
\end{aligned}
$$

Using two inequalities(2.17) and (2.18) above, the following inequality is satisfied

$$
C\left(\operatorname{li}(x)-\frac{x}{\log (x)}\right)<\left(C_{1}-\frac{\sqrt{2 \pi} \alpha}{e}\right) \frac{x}{\log (x)}
$$


10DERIVING VON KOCH'S INEQUALITY WITHOUT USING THE RIEMANN HYPOTHESIS.

According to the assumption(2.8), the positive real number $C>1$ can be choose arbitrary. Hence, choose $C=\sqrt{2 \pi} \alpha / e$ on inequality(2.19) above. Thus, the following condition is satisfied :

$$
\frac{\sqrt{2 \pi} \alpha}{e}\left(\operatorname{li}(x)-\frac{x}{\log (x)}\right)<\left(C_{1}-\frac{\sqrt{2 \pi} \alpha}{e}\right) \frac{x}{\log (x)}
$$

Hence, the following inequality is satisfied :

$$
\frac{\sqrt{2 \pi} \alpha}{e} \operatorname{li}(x)<C_{1} \frac{x}{\log (x)}
$$

The both sides of inequality $(2.21)$ is divided by $\sqrt{2 \pi} \alpha / e$. Therefore, for all real number $\alpha>0$ such that $C_{1}>\sqrt{2 \pi} \alpha / e$, the following inequality is satisfied :

$$
\operatorname{li}(x)<C_{1}\left(\frac{e}{\sqrt{2 \pi} \alpha} \frac{x}{\log (x)}\right)
$$

Namely, using inequality(2.22), for all real number $\alpha>0$ such that $C_{1}>$ $\sqrt{2 \pi} \alpha / e$, there is sufficiently large $x \geq 2$, the following condition is satisfied :

$$
\operatorname{li}(x) \leq \frac{x}{\log (x)} \quad\left(\because C_{1}>\frac{\sqrt{2 \pi} \alpha}{e}\right)
$$

However, the inequality $(2.23)$ contradicts the fact that for sufficiently large $x>$ 2 , the following inequality is satisfied :

$$
\frac{x}{\log (x)}<\operatorname{li}(x) .
$$

Namely, the assumption(2.8) is inconsistent.

Thus, there exist a real number $C>1(\in \mathbb{R})$ such that for all sufficiently large real number $x>2(\in \mathbb{R})$, the following inequality is satisfied:

$$
\pi(x)-\operatorname{li}(x) \leq C\left(\operatorname{li}(x)-\frac{x}{\log (x)}\right)
$$

The end of the case 2) proof.

Consequently, using the discussion Case1) and Case2) above, there exist a positive real number $C>1$ such that for all positive real number $x>2$, the following condition is satisfied :

$$
|\pi(x)-\operatorname{li}(x)| \leq C\left(\operatorname{li}(x)-\frac{x}{\log (x)}\right) .
$$

\subsection{Proof of Theorem(1.9).}


DERIVING VON KOCH'S INEQUALITY WITHOUT USING THE RIEMANN HYPOTHESIS.11

Proof. Using Lemma(1.5) and Lemma(1.8), there exist a positive real number $C>1$ such that for all sufficiently large $x \geq 2$, the following formulas are satisfied :

$$
\begin{aligned}
& |\pi(x)-\operatorname{li}(x)| \\
& \leq C\left(\operatorname{li}(x)-\frac{x}{\log (x)}\right) \quad(\because \operatorname{Lemma}(1.5)) \\
& =C\left(\lim _{\epsilon \rightarrow 0}\left(\int_{0}^{1-\epsilon}+\int_{1+\epsilon}^{x}\right) \frac{d t}{\log (t)}-\left(\left[\frac{t}{\log (t)}\right]_{0}^{1-\epsilon}+\left[\frac{t}{\log (t)}\right]_{1+\epsilon}^{x}\right)\right) \\
& =C\left|\lim _{\epsilon \rightarrow 0}\left(\int_{0}^{1-\epsilon}+\int_{1+\epsilon}^{x}\right) t\left(\frac{1}{\log (t)}\right)^{\prime} d t\right| \quad(\because \text { Partial Integral Formula }) \\
& \leq C\left|\lim _{\epsilon \rightarrow 0}\left(\int_{0}^{1-\epsilon}+\int_{1+\epsilon}^{x}\right) t\left(\frac{\Pi_{\alpha}^{+}(t)}{t}\right)^{\prime} d t\right|(\because \text { Lemma }(1.8)) \\
& =C\left|\int_{0}^{x} t\left(\frac{\Pi_{\alpha}^{+}(t)}{t}\right)^{\prime} d t\right| \\
& =C\left|\int_{0}^{x} t\left(\exp \left(\frac{e}{\sqrt{2 \pi} \alpha}\left(1+\frac{1}{e \log (t)}\right)\right)\right)^{\prime} d t\right| \\
& =C \int_{0}^{x} t\left(\frac{1}{\sqrt{2 \pi} \alpha} \frac{1}{t(\log (t))^{2}} \exp \left(\frac{e}{\sqrt{2 \pi} \alpha}\left(1+\frac{1}{e \log (t)}\right)\right)\right) d t \\
& =C \frac{1}{\sqrt{2 \pi} \alpha} \exp \left(\frac{e}{\sqrt{2 \pi} \alpha}\right) \int_{0}^{x} \frac{1}{(\log (t))^{2}}\left(\exp \left(\frac{1}{\sqrt{2 \pi} \alpha} \frac{1}{\log (t)}\right)\right) d t
\end{aligned}
$$

Put the positive real number $a>0(\in \mathbb{R})$ as follows :

$$
a:=\frac{1}{\sqrt{2 \pi} \alpha}
$$

Using the condition of inequality(1.37), that is, $1 / \sqrt{2 \pi} \leq \alpha$, the positive real number $a>0$ is satisfied as follows :

$$
a \leq 1
$$

Put the variable $v$ as follows :

$$
v:=\frac{a}{\log (t)}
$$

Hence, the following conditions are satisfied :

$$
\begin{aligned}
& d v=\frac{-a d t}{t(\log (t))^{2}} \\
& t=\exp \left(\frac{a}{v}\right)
\end{aligned}
$$


12DERIVING VON KOCH'S INEQUALITY WITHOUT USING THE RIEMANN HYPOTHESIS.

Apply change of variables to the formula(2.27) as follows :

$$
\begin{aligned}
\int_{0}^{x} \frac{1}{(\log (t))^{2}}\left(\exp \left(\frac{1}{\sqrt{2 \pi} \alpha} \frac{1}{\log (t)}\right)\right) d t \\
=\frac{1}{a} \int_{-\infty}^{\frac{a}{\log (x)}}\left|-\exp \left(\frac{a}{v}\right) \exp (v)\right| d v \\
=\frac{1}{a} \int_{-\infty}^{\frac{a}{\log (x)}} \exp \left(\frac{a}{v}\right) \exp (v) d v \\
\leq \frac{1}{a} \int_{-\infty}^{\frac{1}{\log (x)}} \exp \left(\frac{a}{v}\right) \exp (v) d v \quad\left(\because \frac{a}{\log (x)} \leq \frac{1}{\log (x)}\right) \\
\leq \frac{1}{a}\left(\int_{-\infty}^{\frac{1}{\log (x)}} \exp \left(\frac{2 a}{v}\right) d v\right)^{\frac{1}{2}}\left(\int_{-\infty}^{\frac{1}{\log (x)}} \exp (2 v) d v\right)^{\frac{1}{2}} \\
\quad(\because \text { Schwarz inequality) }
\end{aligned}
$$

Next, set the variable $u$ as follows :

$$
u:=\frac{1}{v}
$$

Hence, the following equation is satisfied :

$$
d v=\frac{-d u}{u^{2}}
$$

Apply to change of variables the inside of first bracket on the formula(2.33) as follows :

$$
\begin{aligned}
& \int_{-\infty}^{\frac{1}{\log (x)}} \exp \left(\frac{2 a}{v}\right) d v \\
&=\int_{0}^{\log (x)}\left|\exp (2 a u)\left(-\frac{1}{u^{2}}\right)\right| d u \\
& \leq\left(\int_{0}^{\log (x)} \exp (4 a u) d u\right)^{\frac{1}{2}}\left(\int_{0}^{\log (x)}\left|\frac{1}{u^{4}}\right| d u\right)^{\frac{1}{2}} \\
&(\because \operatorname{Schwarz} \text { inequality }) \\
& \leq\left(\frac{1}{4 a} \exp (4 a \log (x))\right)^{\frac{1}{2}}\left(\frac{1}{3(\log (x))^{3}}\right)^{\frac{1}{2}} \\
&= \frac{1}{2}\left(\frac{1}{a}\right)^{\frac{1}{2}} \exp (2 a \log (x))\left(\frac{1}{3(\log (x))^{3}}\right)^{\frac{1}{2}}
\end{aligned}
$$

Furthermore, the inside of second bracket on the formula(2.33) is satisfied as follows:

$$
\int_{-\infty}^{\frac{1}{\log (x)}} \exp (2 v) d t \leq \frac{1}{2} \exp \left(\frac{2}{\log (x)}\right)
$$


Using formulas(2.33), (2.36) and (2.37), the formula(2.27) is expressed as follows

$$
\begin{aligned}
\mid \pi & (x)-\operatorname{li}(x) \mid \\
\leq & \frac{C}{\sqrt{2 \pi} \alpha} \exp \left(\frac{e}{\sqrt{2 \pi} \alpha}\right) \frac{1}{a} \\
& \cdot\left(\frac{1}{2}\left(\frac{1}{a}\right)^{\frac{1}{2}} \exp (2 a \log (x))\left(\frac{1}{3(\log (x))^{3}}\right)^{\frac{1}{2}}\right)^{\frac{1}{2}}\left(\frac{1}{2} \exp \left(\frac{2}{\log (x)}\right)\right)^{\frac{1}{2}} \\
= & \frac{C}{2}\left(\frac{1}{3 a}\right)^{\frac{1}{4}} \exp \left(\frac{e}{\sqrt{2 \pi} \alpha}\right) \exp (a \log (x))\left(\frac{1}{\log (x)}\right)^{\frac{3}{4}} \exp \left(\frac{1}{\log (x)}\right) \\
= & \frac{C}{2}\left(\frac{1}{3 a}\right)^{\frac{1}{4}} \exp \left(\frac{e}{\sqrt{2 \pi} \alpha}\right) x^{a}\left(\frac{1}{\log (x)}\right)^{\frac{3}{4}} \exp \left(\frac{1}{\log (x)}\right) \\
& \left(\because \exp (a \log (x))=x^{a}\right) \\
= & C\left(\frac{\sqrt{2 \pi} \alpha}{48}\right)^{\frac{1}{4}} \exp \left(\frac{e}{\sqrt{2 \pi} \alpha}\right) x^{\frac{1}{\sqrt{2 \pi} \alpha}}\left(\frac{1}{\log (x)}\right)^{\frac{3}{4}} \exp \left(\frac{1}{\log (x)}\right) \\
& \left(\because a=\frac{1}{\sqrt{2 \pi} \alpha}\right)
\end{aligned}
$$

Consequently, the inequality(1.35) of Theorem(1.9) is satisfied.

\section{Conclisions.}

We derived that Von Koch's inequality without using the Riemann Hypothesis in this paper. Using the fact that Von Koch's inequality is equivalent to the Riemann Hypothesis, we obtained that the Riemann Hypothesis is true. Besides, we obtained a result that the inequality $|\pi(x)-\operatorname{li}(x)| \leq O\left(x^{1 / e} \log (x)\right)$, which has stricter conditions than the Riemann Hypothesis. We further raise that the existence and the value of the limit of upper bound $|\pi(x)-\operatorname{li}(x)|$.

We started this paper to the introduction of the functions $R_{\alpha}^{ \pm}(x)$. We obtained the functions $R_{\alpha}^{ \pm}(x)$ using the concept of Statistical Mechanics. We think that Number theory and Statistical Mechanics are closely related.

\section{Acknowledgments.}

We would like to thank all the people who supported this challenge and to express my deepest respect for giving us the idea. We would like to explain how to derive the distribution functions used in this paper if it gives us an opportunity,

\section{REFERENCES}

[1] Wladyslaw Narkiewicz, The Development of Prime Number Theory, Springer, 2000

[2] P.L.Chebychev. Mémoire sur les nombres premiers. J. de Math. Pures Appl., 17:366-390, 1852

[3] P.L.Chebychev. Sur la totalité des nombres premiers inf érieurs á une limite donneé, J. de Math. Pures Appl., 17:341-365, 1852

[4] Max Planck, Vorlesungen über die Theorie der Wärmestrahlung, J.A Barth, 1906

[5] G. H. Hardy and J. E. Littlewood, Contributions to the theory of the Riemann Zeta-function and the theory of the distribution of primes, Acta Math. 41: 119-196 (1916)

[6] Feynman et.al, The Feynman, Lecture on Physics,Volume III, Quantum Mechanics ,1963 
14DERIVING VON KOCH'S INEQUALITY WITHOUT USING THE RIEMANN HYPOTHESIS.

[7] André LeClair, Riemann Hypothesis and Random Walks: The Zeta Case ,Symmetry 2021, 13,2014

[8] Von Koch H, Sur la distribution des nombres premiers, Acta Mathematica, 24 159-182, 1901

[9] Entropy of Riemann zeta zero sequence, Advanced Modeling and Optimization, Volume 15, Number 2,O. Shanker, 2013

[10] Ioannis Kontoyiannis, Counting the Primes Using Entropy, The 2008 IEEE Information Theory Workshop, Porto, Portugal, Lecture given on Thursday, May 82008

[11] Arturo Ortiz, Hans Henrik Støleum. Studies of entropy measures concerning the gaps of prime numbers, arXiv:1606.08293 [math.GM], 2016.

[12] Michael Atiyah. The Riemann Hypothesis. 2018

[13] Seiji Fujino, Expansion of the Planck's function and Application to Number Theory (In preparation) 